\title{
Modelling of line-profile variability in WC stars
}

\author{
Lydia M. Oskinova ${ }^{1}$, Alexander F. Kholtygin ${ }^{2}$, and F.V. Kostenko ${ }^{2}$ \\ ${ }^{1}$ Department of Physics and Astronomy, University of Glasgow, UK \\ ${ }^{2}$ Astronomical Institute, St. Petersburg University, Russia
}

Suppose that the atmosphere of a WC star is formed by clumps embedded in the homogeneous spherically symmetric stellar wind. A single clump may be modeled as a part of the spherical layer restricted with a surface of a cone. Present the gas density in the cone as a sum of gas density in homogeneous atmosphere and gas density in a clump:

$$
n_{k}^{\mathrm{cl}}(r)=D^{\mathrm{cl}} n_{k}\left(r_{\mathrm{cl}}\right) e^{-\left(\frac{r-r_{\mathrm{cl}}}{\delta r_{\mathrm{cl}}}\right)^{2}}+n_{k}(r) .
$$

Here $n_{k}(r)$ is the gas density in the homogeneous atmosphere, $D^{\mathrm{cl}}$ is the ratio of gas densities at a point $r=r_{\mathrm{cl}}$, and $\delta$ is the size of the clumps in units $r_{\mathrm{cl}}$.

For the law of gas motion in the clump we assume (Kholtygin \& Kostenko 1998):

$$
v_{\mathrm{cl}}(r)=v(r)+\sqrt{2} y e^{0.5-y^{2}} \Delta v_{\mathrm{cl}}, \quad y=\frac{r-r_{\mathrm{cl}}}{\delta r_{\mathrm{cl}}},
$$

Here $\Delta v_{\mathrm{cl}}$ is a free parameter, and $v(r)$ is the $\beta$-velocity law. The profiles of the subordinate $\mathrm{C}$ III $\lambda 5696$ is calculated in SEI-like approximation. Let $F_{i}$ be the clump flux. Then the total contribution of the clumps to the line profile is:

$$
F_{\nu}=2 \pi \int_{0}^{\pi} \int_{M_{\min }}^{M_{\max }} \int_{R_{*}}^{R_{\mathrm{atm}}} N\left(M, r_{\mathrm{cl}}, \theta\right) F_{i}(\nu) d M_{\mathrm{cl}} d r_{\mathrm{cl}} \sin \theta d \theta .
$$

Here $M$ is the mass of a clump, which ranges between $M_{\min }$ and $M_{\max } ; F_{i}$ is the energy emitted by a clump in the frequency interval $d \nu$, and $N$ is the mass spectrum of the clump ensemble. For the outer edge of the atmosphere we adopt $R_{\mathrm{atm}}=50 R_{*}$ supposing that the contribution of the remaining part of the wind in the total line profile is negligible. The shape and variability of the profiles of the $\mathrm{C}$ III lines reflect the properties of the whole clump ensemble.

If we assume a power law for $F_{i}$ :

$$
N\left(F_{i}\right)=A F_{i}^{-\alpha},
$$

where $A$ is the normalizing coefficient, and $\alpha \approx 2.0 \pm 0.4$, then the total flux in the line emitted by a clump is

$$
f_{i}=\int_{-\infty}^{+\infty} I_{i}(\nu) d \nu=\int_{-\infty}^{+\infty} I_{i}^{0} e^{-\frac{1}{2}\left(\frac{\nu-\nu_{i}^{0}}{\sigma_{i}}\right)^{2}} d \nu=I_{i}^{0} \sigma_{i} \sqrt{2 \pi}
$$




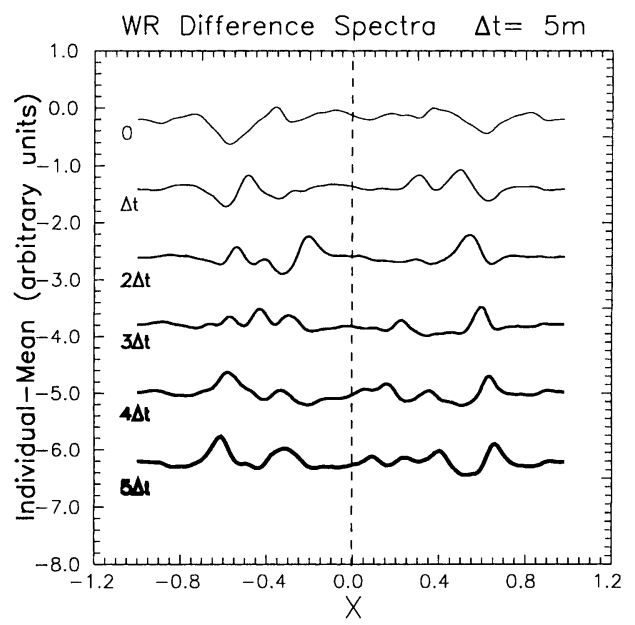

Figure 1. The difference spectra of the $\mathrm{C}$ III $\lambda 5696$ line. $\alpha=1.8$, $F_{\max } / F_{\min }=2 \times 10^{4}, v_{\infty}=2400 \mathrm{~km} \mathrm{~s}^{-1}, \beta=0.5$. The time interval between the sequential spectra $\Delta t=5^{m}$.

which gives

$$
I_{i}^{0}=\frac{f_{i}}{\sigma_{i} \sqrt{2 \pi}}
$$

where $\nu_{i}^{0}$ is the central frequency of the subpeak $i$, and $\sigma_{i}$ is the width of line. Here $f_{i}=F_{i} / F_{\max }$ is the flux normalized to the maximal flux of clumps supposing that each clump form the subpeak with the gaussian profile.

To simulate of the line profile variation we model the whole ensemble of the clumps at the time $t=0$ and suppose that clumps are randomly distributed in cone angle and starting radius and then move independently. The clump parameters are recalculated in the time interval $\Delta t$. The total line profiles $I^{n}(x)$ are evaluated for the moments $t_{n}=n \cdot \Delta t$. We average the different sets of calculated profiles $I^{n}(x)(n=1-M)$, where $M$ is the total number of the profiles and calculate the mean and difference spectra. Resulting difference spectra are given in Figure 1. One can see that the separate scraps of shells produce rapidly moving subpeaks on the emission part of the line profiles which are similar to the observational results (e.g., Moffat et al. 1994). Our modelling revealed that the location of the moving bumps depends strongly on the angle and distance distribution of the clump ensemble, which might be properly adapted for a closer comparison with the observational data. The flux in the individual subpeak depends mainly on the mass of the corresponding clump.

Acknowledgments. This work is funded by the RAS via a RAS/NATO fellowship. AFK and FVK are grateful for the support provided by the Russian Federal Program 'Astronomy'.

\section{References}

Kholtygin, A.F., Kostenko, F.V. 1998, Astrofizika 41, 423

Moffat, A.F.J., Lépine, S., Henriksen, R.N., Robert, C. 1994, ApSS 216, 55 